\title{
Neutrinos as a probe of dark-matter particles
}

\author{
W-Y. Pauchy Hwang 1 \\ Asia Pacific Organization for Cosmology and Particle Astrophysics, \\ Institute of Astrophysics, Center for Theoretical Sciences, \\ and Department of Physics, National Taiwan University, Taipei 106, Taiwan
}

(July 27, 2012)

\begin{abstract}
We try to envision that there might be a dark-matter world and neutrinos, especially the right-handed ones, might be coupled directly with dark-matter particles in the darkmatter world. The candidate model would be the extended Standard Model based on $S U_{c}(3) \times S U_{L}(2) \times U(1) \times S U_{f}(3) \times S U_{R}(2)$, with the search of the detailed version through the aid of the two working rules, "Dirac similarity principle" and "minimum Higgs hypothesis".
\end{abstract}

PACS Indices: 12.60.-i (Models beyond the standard model); 98.80.Bp (Origin and formation of the Universe); 12.10.-g (Unified field theories and models).

\section{Why are neutrinos so interesting?}

Neutrinos have masses, the tiny masses outside the range of the masses of the quarks and charged leptons. This is one of the most important experimental results over the last thirty years. What are the theoretical implications of this fact?

If we take some minimal extension of the Standard-Model group, that neutrinos have tiny masses could be taken as a signature that there is a heavy extra $Z^{\prime 0}$, so that a new Higgs doublet should exist. This extra $Z^{\prime 0}$ then requires the new "remote" Higgs doublet[1]. This Higgs doublet also generates the tiny neutrino masses. This is the $S U_{c}(3) \times S U_{L}(2) \times$ $U(1) \times U(1)$ extension. Is this true?

However, there is room left for something very interesting. Remember that the righthanded neutrinos never enter in the construction of the minimal Standard Model [2]. The message that the right-handed neutrinos seem to be "unwanted" could be telling us something. Now, the fact that neutrinos have tiny masses suggests that "more naturally" they would be four-component Dirac particles, and maybe unlikely to be the two-component Majorana particles.

Now, the right-handed neutrinos are "unwanted" in the minimal Standard Model, leaving the room so that they could form some multiplet under a new gauge group (beyond the minimal Standard Model). In fact, we have some candidate from the symmetries - the family symmetry that there are three generations in the building blocks of ordinary matter, and so far only three. We have seen this fact, but we don't know why - let's speculate that it could be the story associated with the dark-matter world.

Thus, it arises naturally the so-called family gauge theory [3]. Note that the right-handed neutrinos do not appear in the minimal Standard Model. Indeed, we could make a massive $S U_{f}(3)$ gauge theory completely independent of the minimal Standard Model, including

\footnotetext{
${ }^{1}$ Correspondence Author; Email: wyhwang@phys.ntu.edu.tw; arXiv:xxxx (hep-ph, to be submitted)
} 
the particle content. We could treat $\left(\nu_{\tau R}, \nu_{\mu R}, \nu_{e R}\right)$ as a triplet under this $S U_{f}(3)$ - so to give rise to a family gauge theory. Because the anomaly might not hurt, we could drop the right-handed labels from the neutrinos. This completes the derivation of the family gauge theory [3]. The $S U_{f}(3)$ is by definition the massive gauge theory all the involved particles are dark-matter particles in the dark-matter world.

Basically, we could try to combine the minimal Standard Model $S U_{c}(3) \times S U_{L}(2) \times U(1)$ with $S U_{f}(3)$, with $\left(\nu_{\tau R}, \nu_{\mu R}, \nu_{e R}\right)$ the basic $S U_{f}(3)$ triplet. Here $S U_{f}(3)$ has an orthogonal neutrino multiplet since the right-handed neutrinos do not enter at all the minimal Standard Model. In this way, we obtain the $S U_{c}(3) \times S U_{L}(2) \times U(1) \times S U_{f}(3)$ minimal model. Or, the right-handed indices could be dropped altogether in the family group, just like the other $S U_{c}(3)$ combining with $S U_{L}(2) \times U(1)$ as far as anomalies are concerned.

In this case [3, the three family calls for $S U_{f}(3)$ and to make the gauge bosons all massive the minimum choice would be a pair of complex Higgs triplets - apparently a kind of broken gauge symmetry. The scenario is such that the eight gauge bosons and the four left-over Higgs particles all have masses greater than a few $\mathrm{TeV}^{\prime} s$ (i.e. above the LHC energies). The reason why they all should be massive is that they can enter the loop diagrams in the ordinary-matter world (i.e., the world initially described by the minimal Standard Model). At this point, it seems that there is some sort of "minimum Higgs hypothesis", after forty years of searching for the Higgs particles. Under the "minimum Higgs hypothesis", the structure of the underlying Higgs mechanism is pretty much determined. Then, neutrinos acquire their masses, to the leading order, with the aid of both the Higgs triplets. In addition, the loop diagrams involving the gauge bosons (familons) also contribute to neutrino masses. The dark-matter origins of neutrino masses may explain why they are so tiny.

We know that there are three generations of quarks and leptons but don't know exactly why. This may be a symmetry that we have already "seen", but not in details. In view of the existing structure of the minimal Standard Model, we suspect that most symmetries might be realized in the form of gauge theories (our own prejudice), in this case a family gauge theory [3]. On other hand, the missing right-handed sector is always a mystery to many of us. So, the next option is to employ the notion originated by Pati and Salam [4] that the left-right symmetry is restored at some even higher energy. Again, the Higgs sector is determined via "minimum Higgs hypothesis"; otherwise, there are too many choices as originally proposed 4, even if the Higgs sector is concerned.

We could move one step forward. We see that the three generations are already there (even though we have not seen the feeble interactions so far). Judging from the energies which we could reach at $\mathrm{LHC}$, we could set the mass scale at a few $\mathrm{TeV}^{\prime} s$ (for familons and family Higgs). On the other hand, until the LHC energies we haven't seen any signature that the right-handed sector would be back - so setting the scale to at least hundreds of $T e V^{\prime} s$. In other words, we are talking about the extended Standard Model based on the group $S U_{c}(3) \times S U_{L}(2) \times U(1) \times S U_{f}(3) \times S U_{R}(2)$.

To summarize, we think that, when we talk about jointly the ordinary-matter world and the dark-matter world, the family symmetry is there (explaining why there are three generations of fermions) and the missing right-handed sector, though still not there, would eventually come back (as energies increase). Everything is written through the Standard-Model way, or in the gauge-field fashion. It is naively renormalizable (i.e., by power counting). 
It is clear that the minimum extended Standard Model would be the extended Standard Model to be based on the group $S U_{c}(3) \times S U_{L}(2) \times U(1) \times S U_{f}(3) \times S U_{R}(2)$. That is our rationale.

Maybe in the following we would like to append two lengthy remarks - first, on the possible existence of dark-matter galaxies; and, second, on the validity of the two working rules, "Dirac similarity principle" and "minimum Higgs hypothesis".

We would be curious about how the dark-matter world looks like, though it is difficult to verify experimentally. The first question would be: The dark-matter world, $25 \%$ of the current Universe (in comparison, only $5 \%$ in the ordinary matter), would clusterize to form the dark-matter galaxies, maybe even before the ordinary-matter galaxies. The dark-matter galaxies would then play the hosts of (visible) ordinary-matter galaxies, like our own galaxy, the Milky Way. Note that a dark-matter galaxy is by our definition a galaxy that does not possess any ordinary strong and electromagnetic interactions (with our visible ordinary-matter world), i.e. that consists of dark-matter particles only. This fundamental question deserves some thoughts, for the structural formation of our Universe.

In the ordinary-matter world, strong and electromagnetic forces make the clustering a very different story - they manufacture atoms, molecules, complex molecules, and chunks of matter, and then the stars and the galaxies; the so-called "seeded clusterings". The relatively-rapid story up to chunks of matter is mainly due to the residual electromagnetic and strong forces, rather than from gravitational forces. On the other hand, the seeds in the dark-matter world could come from relatively-stable extra-heavy dark-matter particles (such as familons and family Higgs, depending on how many decay channels) - one such particle would be equivalent to thousands of ordinary-matter molecules. Note that, in the ordinary-matter world, the sequence of atoms-molecules-complex molecules-etc., up to the mass of the $\mathrm{TeV}$ level, yields the "seeds" of the clusterings - the seeded clustering that is relevant for the time span (in about 1 Gyr) of our young Universe in the ordinary-matter world. In our extended Standard Model, such seeds for dark-matter galaxies might come from relatively-stable heavy dark-matter particles (with the mass greater than, e.g., a few $T e V)$. They live relatively long in the absence of decay channels.

The idea of the "seeded" clustering, and its potential importance in galactic formation and evolution is rather intuitive - hence intuitively acceptable. However, this idea is at odd with the standard wisdom that the gravitational force be solely responsible for clusterings - and for galactic formation as well. To determine which one is correct for the time span of about 1 Gyr (i.e. the age of our young Universe), serious numerical simulations may be needed.

On the other hand, it would be too broad to identify which extended Standard Model, in detail, could be the final choice. With the "minimum Higgs hypothesis" [5], the Higgs sector is essentially fixed once the group $S U_{c}(3) \times S U_{L}(2) \times U(1) \times G$ with $G$ the extension is fixed. On the other hand, the "Dirac Similarity Principle" helps to fix the particle contents. With these working rules [5] that are in essence used for several decades, the search for the correct extended Standard Model could be sharpened and thus much easier.

Or, we could construct so many extended Standard Models, even if the gauge group is fixed to be, e.g., $S U_{c}(3) \times S U_{L}(2) \times U(1) \times S U_{f}(3) \times S U_{R}(2)$ - due to the unknown Higgs multiplets and due to whether the fermions would be Dirac particles and, furthermore, the standard-model multiplets would be the best choice. Using "Dirac similarity principle" 
and "minimum Higgs hypothesis" [5], we proceed to make choices only those "naively" renormalizable extended Standard Model(s) and to test its validity.

To push forward the "final" Standard Model, we should have a comprehensive successful phenomenology [6]. On the $S U_{c}(3) \times S U_{L}(2) \times U(1) \times S U_{f}(3) \times S U_{R}(2)$ extended Standard Model, the first goal is to pin down the manifestations of the $S U_{f}(3)$ gauge sector. The physics of the neutrino sector gets modified and the $\tau-\mu-e$ universality is in principle no longer there, depending on the strength of the $S U_{f}(3)$ coupling. The reason for the neutrino couplings is that the neutrino is only species in the ordinary-matter world that acts also as dark matter. And if the $S U_{f}(3)$ gauge sector is there, its communication with us (the ordinary matter) is only through the neutrinos. So, the breakdown of the $\tau-\mu-e$ universality, albeit it could be very small, is absolutely crucial.

In the said family gauge theory, we introduce a pair of complex Higgs triplets in order to guarantee that all gauge bosons ("familons") and the remaining four Higgs particles are massive, say, greater than a few $\mathrm{TeV}$. They have to be massive mainly because, if massless, the loop diagrams involving these dark-matter particles could become dominant. We also note that the pair of complex Higgs triplets and the neutrino triplet can form a singlet, the off-diagonal neutrino mass interaction, providing a natural mechanism for neutrino oscillations.

\section{Where could we see the detailed tests?}

Neutrino oscillations are firmly established, though much more yet to come; this is where $\nu_{e}$ suddenly reappears as $\nu_{\mu}$ or as $\nu_{\tau}$, where $\nu_{\mu}$ as $\nu_{\tau}$ or as $\nu_{e}$, and so on. This is precisely the lepton-flavor-violating interaction as given by

$$
i \eta \bar{\Psi} \cdot\left\{\Phi_{+}+\epsilon \Phi_{-}\right\} \times \Psi
$$

with $\Phi_{ \pm}$the pair of complex Higgs triplets and $\Psi$ the neutrino triplet $\left(\nu_{\tau}, \nu_{\mu}, \nu_{e}\right)$. Here the remaining Higgs particles are massive and the associated vacuum expectation values are given by $u_{ \pm}[3]$. The role of this off-diagonal interaction, a lepton-flavor-violating interaction, may be underestimated.

The other important possibilities come from the serious search for lepton-flavor-violating decays (reactions) of charged leptons, such as $\mu \rightarrow e+\gamma, \mu \rightarrow 3 e, \mu+A \rightarrow e+A^{*}$, and so on. For $\mu \rightarrow e+\gamma$ as example, we show in Figs. 1(a), 1(b), and 1(c) that the off-diagonal interaction is in operation. Using a recent calculation [7], it is reduced to an effective interaction as given by

$$
\begin{gathered}
T=2 \cdot \frac{G_{F}}{\sqrt{2}} \quad \cdot \eta\left(u_{+}+\epsilon u_{-}\right) \cdot\left(m_{1}+m_{2}\right) \cdot(+2 i) \frac{e}{(4 \pi)^{2}} \\
\cdot \bar{u}\left(p^{\prime}, s^{\prime}\right) \frac{\gamma \cdot \epsilon}{\sqrt{2 k_{0}}}\left(1+\gamma_{5}\right) u(p, s) .
\end{gathered}
$$

The decay rate is determined by $d \Gamma=\frac{d^{3} p^{\prime}}{(2 \pi)^{3}} \frac{d^{3} k}{(2 \pi)^{3}}(2 \pi)^{4} \delta^{4}\left(p-p^{\prime}-k\right) \frac{1}{2} \sum|T|^{2}$, yielding the branching ratio proportional to $O\left(m^{4} / m_{\mu}^{4}\right)$. The tininess of the neutrino masses makes the observation virtually impossible. 


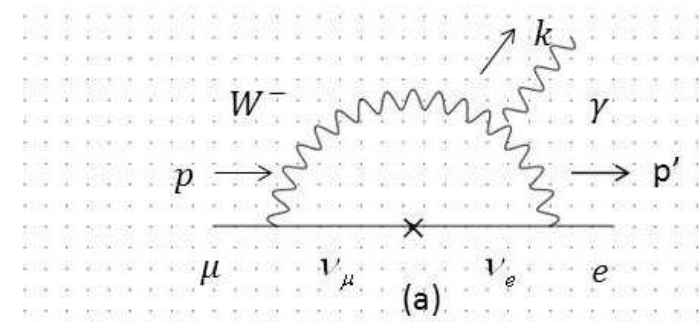

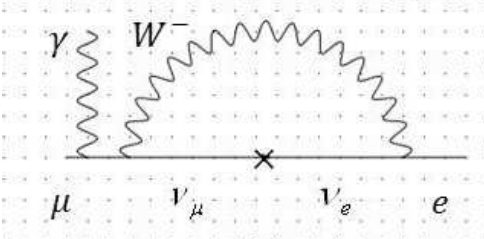

(b)

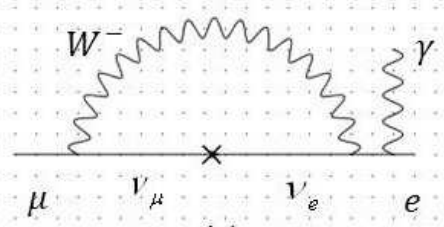

(c)

Figure 1: The leading diagrams for $\mu \rightarrow e+\gamma$.

Maybe the other possibility is through the detailed tests of the $\tau-\mu-e$ universality. Years ago, this universality entered the arguments in the $\tau$-mass measurements. We need to re-check the arguments in view of the present tiny deviations. In addition, the tests of the Standard Model involve the so-called $\rho$ parameter - to check the $\mu-e$ universality, we could compare the reactions induced by $\nu_{\mu}$ with those induced by $\nu_{e}$. It is clear that a new field is opening up.

\section{Episode}

In writing up this note, I have kept in mind that the spiral tail of the Milky Way is caused by the dark-matter aggregate, or even a dark-matter galaxy, of four or five times the mass of the Milky Way, and similarly for other spiral galaxies. In the fronts of astrophysics, further investigations of these spiral galaxies may eventually offer a lot more in the dark-matter world.

Of course, we should remind ourselves that, in our ordinary-matter world, those quarks can aggregate in no time, to hadrons, including nuclei, and the electrons serve to neutralize the charges also in no time. Then atoms, molecules, complex molecules, and so on. These serve as the seeds for the clusters, and then stars, and then galaxies, maybe in a time span of 1 Gyr (i.e., the age of our young Universe). The aggregation caused by strong and electromagnetic forces is fast enough to help giving rise to galaxies in a time span of 1 Gyr. On the other hand, the seeded clusterings might proceed with abundance of extra-heavy dark-matter particles such as familons and family Higgs, all greater than a few $\mathrm{TeV}$ and with relatively long lifetimes (owing to very limited decay channels). So, further simulations on galactic formation and evolution may yield clues on our problem.

Finally, coming back to the fronts of particle physics, neutrinos, especially the righthanded neutrinos, might couple to the dark-matter particles. Any further investigation along this direction would be of utmost importance. It may shed light on the nature of the dark-matter world. 


\section{Acknowledgments}

This research is supported in part by National Science Council project (NSC 99-2112-M002-009-MY3). We wish to thank the authors of the following books [2] for thorough reviews of the minimal Standard Model.

\section{References}

[1] W-Y. P. Hwang, arXiv:1009.1954v2 (hep-ph, 27 May 2011); W-Y. P. Hwang, Phys. Rev. D36, 261 (1987); see the second paper for many more earlier references.

[2] Ta-You Wu and W-Y. Pauchy Hwang, "Relatistic Quantum Mechanics and Quantum Fields" (World Scientific 1991); Francis Halzen and Alan D. Martin, "Quarks and Leptons" (John Wiley and Sons, Inc. 1984); E.D. Commins and P.H. Bucksbaum, "Weak Interactions of Leptons and Quarks" (Cambridge University Press 1983). This was "my" early list of the textbooks on the "Standard Model".

[3] W-Y. Pauchy Hwang, Nucl. Phys. A844, 40c (2010); W-Y. Pauchy Hwang, International J. Mod. Phys. A24, 3366 (2009); the idea first appeared in hep-ph, arXiv: 0808.2091; talk presented at 2008 CosPA Symposium (Pohang, Korea, October 2008), Intern. J. Mod. Phys. Conf. Series 1, 5 (2011); plenary talk at the 3rd International Meeting on Frontiers of Physics, 12-16 January 2009, Kuala Lumpur, Malaysia, published in American Institute of Physics 978-0-7354-0687-2/09, pp. 25-30 (2009).

[4] J.C. Pati and A. Salam, Phys. Rev. D10, 275 (1974); R.N. Mohapatra and J.C. Pati, Phys. Rev. D11, 566 (1975); D11, 2559 (1975).

[5] W-Y. P. Hwang, arXiv:11070156v1 (hep-ph, 1 Jul 2011), Plenary talk given at the 10th International Conference on Low Energy Antiproton Physics (Vancouver, Canada, April 27 - May 1, 2011), to be published.

[6] Particle Data Group, "Review of Particle Physics", J. Phys. G: Nucl. Part. Phys. 37, 1 (2010).

[7] W-Y. Pauchy Hwang, arXiv:1207.6443v1 [hep-ph] 27 July 2012. 